\title{
INFECÇÕES EM DISPOSITIVOS NEUROLÓGICOS IMPLANTÁVEIS EM CRIANÇAS E ADOLESCENTES
}

\author{
Márcia Maria Macêdo Lima ${ }^{1}$, Carlos Umberto Pereira², Angela Maria Silva ${ }^{3}$
}

\begin{abstract}
RESUMO - Objetivo: Determinar a freqüência, as causas, o sítio específico e as manifestações clínicas e laboratoriais das infecções em crianças e adolescentes após a implantação de dispositivos neurológicos (DVP) no Hospital Governador João Alves Filho (Aracaju SE). Método: Estudo prospectivo, observacional, não controlado de 50 pacientes, submetidos a DVP (58 procedimentos), no período de janeiro de 2003 a outubro de 2004. Resultados: Observaram-se taxas de infecção por procedimento de $27,6 \%$, taxas de infecção de índice cirúrgico zero, 1 e 2 de $25,7 \%$ e 30,4\%, respectivamente (NNIS-CDC). A infecção de sítio cirúrgico foi a principal complicação com $50 \%$ das infecções. Conclusão: Taxa de infecção por procedimento, paciente e índice de risco cirúrgico mostraram-se elevadas. Não houve significância estatística com relação à idade, etiologia da hidrocefalia, ao tipo de procedimento (derivação primária e reinserção), tempo de internação pré-operatória, duração da cirurgia, antibioticoprofilaxia, cateter SNC prévio e índice de risco cirúrgico.
\end{abstract}

PALAVRAS-CHAVE: hidrocefalia, derivação ventrículoperitoneal, infecção em sistema de derivação ventrículoperitoneal.

\begin{abstract}
Ventriculoperitoneal shunt infections in children and adolescents with hydrocephalus
ABSTRACT - Objective: To determine frequency, etiology, site and clinical and laboratory findings of ventriculoperitoneal shunt (VPS) infections in children and adolescents with hydrocephalus managed in Hospital Governador João Alves Filho, Aracaju SE, Brazil. Method: A non-controlled prospective observational study comprising 50 patients that underwent VPS (58 procedures) from January/2003 to October/2004. Results: Infection rate per procedure was $27.6 \%$; surgical risk index (NNISS-CDC) 0 and 12 were $25.7 \%$ and $30.4 \%$ respectively; surgical site infection was the main complication with $50 \%$ of the cases. Conclusion: Infection rates per procedure, per patient, and per surgical risk index were high. No statistical differences were found related to the following: age, etiology of hydrocephalus, type of procedure, pre-operative length of stay, duration of procedure, antibiotic prophylaxis, previous central nervous system catheter, and surgical risk index.
\end{abstract}

KEY WORDS: hydrocephalus, ventriculoperitoneal shunt, ventriculoperitoneal shunt infections.

O tratamento cirúrgico da hidrocefalia através da derivação ventrículo peritoneal (DVP) tem na infecção a maior causa de morbidade, com graves repercussões como déficit intelectual, neurológico e cognitivo ${ }^{1,2}$. Diversos fatores de risco contribuem para infecção em DVP, tais como: causa da hidrocefalia, recém nascidos, inserção primária do sistema de derivação, meningite prévia, inadequada habilidade técnica da equipe cirúrgica, tempo cirúrgico longo, presença de fístula liquórica, necrose de couro cabeludo e perfu- ração ou infecção de vísceras abdominais ${ }^{3-5}$. Os microrganismos mais prevalentes são os Sthaphylococcus aureus (12-40\%) e Sthaphylococcus epidermidis (52,8$88,9 \%)$. Entre os bacilos Gram-negativos (9-22\%), são freqüentes as Enterobactérias, Klebsiella pneumoniae e Pseudomonas aeruginosa ${ }^{1,6,7}$.

Mundialmente, as taxas de infecção variam de 0 a $31,4 \%$, com média de 10 a $15 \%{ }^{8-10}$. Dados de infecção de sítio cirúrgico por procedimento e categoria de risco de acordo com a metodologia NNIS (National

Hospital Governador João Alves Filho, Aracaju SE, Brasil e NPGME/Universidade Federal de Sergipe, Aracaju SE, Brasil: ${ }^{1}$ Mestre, Infectologista do Hospital Governador João Alves Filho e do HU/Universidade Federal de Sergipe; ${ }^{2}$ Doutor, Professor Adjunto do CCBS/Universidade Federal de Sergipe e Neurocirurgião do Hospital Governador João Alves Filho; ${ }^{3}$ Doutora, Professora Adjunta do CCBS/ Universidade Federal de Sergipe e Infectologista do Hospital Governador João Alves Filho.

Recebido 9 Junho 2006, recebido na forma final 14 Setembro 2006. Aceito 31 Outubro 2006. 
Nosocomial Infection Surveillance System) - CDC (Centers for Disease Control and Prevention) - USA, de 1992-2004, demonstraram taxa de 4,42\% para índice de risco cirúrgico zero e de 5,36\% para índice de risco cirúrgico 1, 2 e 3 para os procedimentos de inserção de DVP ${ }^{11}$.

Este estudo tem por objetivo determinar a freqüência, as causas, o sítio específico e as manifestações clínicas e laboratoriais de infecção após a implantação de dispositivos neurológicos em crianças e adolescentes no Hospital Governador João Alves Filho (Aracaju-SE).

\section{MÉTODO}

Realizou-se estudo prospectivo, observacional, não controlado de 50 pacientes de 0 a 17 anos, de janeiro/2003 a outubro/2004, submetidos a DVP.

Levantaram-se dados de prontuários, como história de meningites prévias, número de cirurgias anteriores para colocação de cateter no sistema nervoso central (SNC), gênero, idade, causa da hidrocefalia, número de dias de internação pré-operatória, exame de líquido cefalorraquidiano (LCR), data e duração da cirurgia, classificação préoperatória de gravidade do paciente - ASA (American Society of Anesthesiologists), classificação do procedimento operatório de acordo com o potencial de contaminação (limpa, potencialmente contaminada, contaminada e infectada) $)^{12}$, uso de antimicrobiano profilático e o procedimento cirúrgico realizado.

O risco de infecção de sítio cirúrgico foi determinado de acordo com o método $\mathrm{NNIS}^{13}$ no qual o risco varia de 0 a 3, de acordo com a presença ou não dos fatores: ferida contaminada ou infectada; duração da cirurgia acima do tempo previsto daquele determinado pelo percentil 75 , obtido na distribuição da duração cirúrgica para aquele procedimento (em derivação do SNC o tempo limite é de 2 horas); classificação pelo índice ASA pré-operatória III, IV ou V. As operações terão, conseqüentemente, zero ponto, quando nenhum fator de risco estiver presente até o máximo de três pontos, quando todos estiverem presentes ${ }^{14,15}$.

A média de risco de infecção cirúrgica (ARIC) foi obtida através da média de fatores de risco presentes no grupo de pacientes com sistema DVP, dividindo-se a soma dos fatores de risco de todos os pacientes pelo total de pacientes. Para o ajuste da taxa de infecção de sítio cirúrgico (ISC) pelo risco, dividiu-se a taxa obtida pelo $\mathrm{ARIC}^{13}$.

No pós-operatório procurou-se avaliar a presença de infecção de sítio cirúrgico, meningite, peritonite, sepsis clínica e bacteremia de acordo com os critérios do NNIS. O seguimento dos pacientes após a alta foi realizado através de visitas domiciliares e/ou contatos telefônicos. Foram questionados estado físico e cicatrização de ferida operatória.

As variáveis foram descritas através de freqüências absolutas e relativas, média, desvio padrão. Os resultados foram testados empregando o método do qui-quadrado $\left(\chi^{2}\right)$ corrigido de Yates, o teste exato de Fischer e o risco relativo (RR). Os limites de confiança $95 \%$ foram calculados no programa EPI INFO 2004.

\section{RESULTADOS}

A população em estudo compreendeu 50 pacientes com idade variando de 0 a 204 meses, com média de 31 meses; 20 (40\%) pacientes tinham idade menor que seis meses; 27 (54,0\%) do gênero masculino e 23 $(46,0 \%)$ do gênero feminino, submetidas a 58 procedimentos cirúrgicos.

Ao se analisar a relação entre número de procedimentos de sistema DVP e idade ( $>$ ou $<6$ meses), entre etiologia de hidrocefalia congênita e não congênita (adquirida e desconhecida), entre os tipos de procedimentos realizados (derivação de LCR primária e inserção secundária), entre cateter prévio de SNC (>5 dias) e infecção, entre o uso ou não do antimicrobiano profilático, tempo de cirurgia (ponto de corte $>$ ou $\leq 2$ horas) e categoria de risco cirúrgico (baixo (0), intermediário (1) e alto (2)), os resultados não mostraram diferenças estatísticas, como descritos na Tabela 1 .

Trinta e cinco $(60,3 \%)$ dos 58 procedimentos foram classificados no índice cirúrgico zero, 22 no índice de risco cirúrgico $1(38,0 \%-22 / 58)$ e um no índice de risco cirúrgico 2 (1,7\%-1/58). Em 98,3\% (57/58) dos procedimentos o índice de risco foi baixo e intermediário (0 e 1). Apenas um procedimento foi realizado no índice de risco alto (2) em um RN prematuro de 28 semanas. A média de risco de infecção cirúrgica foi 0,41 por procedimento (24/58). A taxa ajustada de infecção cirúrgica pelo ARIC ficou em 58,8\% $(24,1 / 0,41)$.

Ocorreram 16 infecções após implantação do sistema DVP em $13(26,0 \%)$ dos 50 pacientes, o que perfaz uma taxa de infecção de $32,0 \%$ por paciente. As demais taxas e sítios de infecções observadas estão descritas na Tabela 2.

Ao se selecionar apenas os casos de cirurgias com ou sem ISC (ISC superficial, meningite, peritonite) e ao se avaliar a ocorrência de infecções de sítio cirúrgico que podia ser explicada pelos fatores de risco (baixo (0) e intermediário (1) e alto (2)), não se evidenciou associação entre categoria de risco e número de cirurgias com ou sem infecção (Tabela 3).

Nos cinco casos de meningite após DVP apenas em dois houve confirmação de cultura positiva (Proteus mirabilis; Streptococos pneumoniae), e em um deles ocorreu positividade na Prova do Látex (Pneumococo). A hemocultura foi positiva para Sthaphylococcus epidermidis em um dos oito casos de ISC. 
Tabela 1. Procedimentos, $n^{\circ}$ de infecções e análise estatística.

\begin{tabular}{|c|c|c|}
\hline Características & $\%$ ( $\mathrm{n}^{\circ}$ infecções/procedimentos) & Análise estatística \\
\hline Idade & & $\chi^{2}$ (Yates) $=0,009 ; p=0,926$ \\
\hline$\leq 6$ meses & $30,4(7 / 23)$ & \\
\hline$>6$ meses & $25,7(9 / 35)$ & \\
\hline Etiologia da hidrocefalia & & $\chi^{2}$ (Yates $)=0,032 ; p=0,858$ \\
\hline Congênita & $27,0(10 / 37)$ & \\
\hline Não congênita & $28,6(6 / 21)$ & \\
\hline Indicação do procedimento & & $\chi^{2}$ (Yates $)=0,949 ; p=0,330$ \\
\hline Primária & $22,5(9 / 40)$ & \\
\hline Secundária & $38,9(7 / 18)$ & \\
\hline Cateter SNC $>5$ dias & & $\chi^{2}$ (Yates) $=0,835 ; p=0,361$ \\
\hline Sim & $40,0(6 / 15)$ & \\
\hline Não & $23,2(10 / 43)$ & \\
\hline ATM profilático & & $\mathrm{p}=0,567$ (Fischer) \\
\hline Sim & $28,3(13 / 46)$ & \\
\hline Não & $25,0(3 / 12)$ & \\
\hline Tempo de cirurgia & & $R R=1,09 ; I C \quad 95 \%=0,6-2,0$ \\
\hline$>2$ horas & $33,3(2 / 6)$ & \\
\hline$\geq 2$ horas & $26,9(14 / 52)$ & \\
\hline Categoria de risco cirúrgico & & $\mathrm{RR}=1,07 ; \mathrm{IC} 95 \%=0,76-1,49$ \\
\hline Baixo (0) & $25,7(9 / 35)$ & \\
\hline Intermediário (1) e Alto (2) & $30,4(7 / 23)$ & \\
\hline
\end{tabular}

SNC, sistema nervoso central; ATM, antimicrobiano.

Tabela 2. Taxas e sítio de infecção em DVP.

\begin{tabular}{lc}
\hline Características & $\begin{array}{c}\% \text { ( }{ }^{\circ} \text { infecções/ } \\
\text { procedimentos) }\end{array}$ \\
\hline Taxa de infecção em DVP & $27,6(16 / 58)$ \\
Taxa de ISC (superficial, meningite, & $24,1(14 / 58)$ \\
peritonite) & \\
Taxa de infecção em cirurgia limpa & $24,5(13 / 53)$ \\
Infecção em paciente c/ meningite prévia & $25,0(2 / 8)$ \\
& \\
Sítio de infecção & \\
$\quad$ Infecção de sítio cirúrgico superficial & $50,0(8 / 16)$ \\
$\quad$ Meningite & $31,2(5 / 16)$ \\
Peritonite & $6,3(1 / 16)$ \\
Bacteremia & $12,5(2 / 16)$ \\
\hline
\end{tabular}

ISC, infecção de sítio cirúrgico; DVP, derivação ventrículo peritoneal.

Tabela 3. Número de cirurgias com ou sem ISC de acordo com a categoria de risco.

\begin{tabular}{lcc}
\hline Categoria de risco & $\begin{array}{c}\mathrm{N}^{\circ} \text { de cirurgias } \\
\text { com ISC }\end{array}$ & $\begin{array}{c}\mathrm{N}^{\circ} \text { de cirurgias } \\
\text { sem ISC }\end{array}$ \\
\hline Baixo (0) & $08 / 14$ & $27 / 44$ \\
Intermediário e & $06 / 14$ & $17 / 44$ \\
Alto (1) (2) & \\
\hline $\mathrm{RR}=1,04$ IC 95\%=[0,77-1,41] $\mathrm{p}=0,974 ;$ ISC infecção de sítio cirúrgico
\end{tabular}

Nos dois pacientes com bacteremia, houve crescimento de Enterococos faecalis + fungos e Enterobacter cloacae + fungos, respectivamente.

As manifestações clínicas foram febre $\left(\geq 38^{\circ} \mathrm{C}\right)$ (81,3\%-13/16), convulsões 56,3\% (9/16), irritabilidade e alteração do nível de consciência (62,3\%-10/16), dor abdominal $(25,0 \%-4 / 16)$, hiperemia no trajeto do cateter (37,5\%-6/16), dor no local da inserção do cateter e secreção no sítio cirúrgico $(50 \%-8 / 16)$.

O tempo médio do diagnóstico de infecção foi 26,7 dias (13/16-81,3\%). Treze infecções $(81,3 \%)$ foram diagnosticadas em menos de um mês após o procedimento operatório (4 casos de meningite, 1 de peritonite, 6 de ISC superficial e 2 de bacteremia) e 3 infecções $(18,7 \%)$ entre 1 e 4 meses ( 1 caso de meningite e 2 de ISC superficial).

Dos 50 pacientes acompanhados no período de três a 540 dias de pós-operatório (média 186 dias), $34(68 \%)$ foram contatados e estavam bem na data do seguimento. Dos 16 pacientes que apresentaram infecção de sistema DVP seis (37,5\%) evoluíram para o óbito, dos quais três por meningite e dois por bacteremia. O sexto caso apresentou ISC superficial, no entanto a causa do óbito não foi devida à infecção do cateter, mas decorrente de hipertensão intracra- 
niana. A taxa de mortalidade relacionada à falência por infecção em sistema de DVP foi 10\% (5/50) e a taxa de letalidade foi 31,3\% (5/16).

\section{DISCUSSÃO}

O tratamento da hidrocefalia através da DVP tem nas complicações infecciosas um persistente desafio a vencer. Um dos mais importantes fatores em infecções de sistema de DVP é a idade. Diversos autores $^{9,16,17}$ relataram forte associação entre menores que seis meses e infecção, numa freqüência de 2,6 vezes maior que maiores de um ano. Outros estudos ${ }^{18,19}$ não encontraram diferenças entre taxa de infecção e diferentes grupos de idade do paciente. Em nosso estudo, o percentual de infecções foi discretamente maior (30,4\%-7/23) entre os menores que seis meses com relação aos maiores que seis meses de idade (25,7\%-9/35); entretanto, não houve significância estatística $(p=0,926)$.

A distribuição etária tem relação com as causas de hidrocefalia, pois a maioria das crianças tem seu diagnóstico em torno dos seis meses de vida. Enger et al. ${ }^{17}$ encontraram taxa elevada $(15,1 \%)$ de infecção em sistema de DVP em crianças com mielomeningocele, mas não conclui se houve relação com a idade ( $<6$ meses). Em concordância com outros autores ${ }^{8,20}$, o nosso estudo não encontrou qualquer diferença significante nas taxas de infecção e as diferentes etiologias de hidrocefalia $(p=0,86)$.

Em algumas séries ${ }^{8,16}$, não houve diferenças nas taxas de infecção entre o procedimento inicial e revisão do sistema de DVP, o que está em concordância com nossos estudos $(p=0,330)$. Entretanto, Enger et al. ${ }^{17}$, mostraram um aumento no risco de infecção liquórica após DVP em inserções primárias do que em revisões, relacionando com idade mais precoce no momento do procedimento, tempo cirúrgico mais longo, maior exposição da pele e de contatos acidentais com o cateter.

A presença de cateter em SNC prévio ao novo procedimento cirúrgico é considerada fator de risco que predispõe a meningite ${ }^{21}$. Kulkarni et al. ${ }^{3}$, no entanto, não demonstraram significância estatística nos seus estudos. Na nossa observação, das 15 crianças com cateter prévio submetidas a DVP, 40\% (6/15) evoluíram com infecção pós-operatória, e em três foi diagnosticada meningite, no entanto sem significância estatística $(p=0,361)$, apesar da importância clínica.

A antibioticoprofilaxia em neurocirurgia para cateter de sistema de DVP, ainda é controversa, com benefícios não estabelecidos ${ }^{22}$, mas alguns autores recomendam o seu uso ${ }^{23,24}$. A profilaxia observada em nosso estudo foi administrada em 79,3\% (46/58) dos casos, mas seu uso não apresentou diferença estatística $(p=0,567)$ com relação ao número de infecções pós-operatórias.

Os estudos de Kulkarni et al. ${ }^{3}$ descreveram média de 47,9 22,3 minutos, na duração dos procedimentos. O tempo cirúrgico não foi considerado significante estatisticamente como fator de risco para infecção de sistema de DVP por outros autores ${ }^{8,18}$. A duração dos procedimentos observada em nosso estudo teve uma média de $83,7 \pm 34,2$ minutos, denotando um tempo cirúrgico prolongado. Ao se agrupar os procedimentos de acordo com o ponto de corte de $2 \mathrm{~h}$ que corresponde ao percentil 75 da metodologia NNIS, os resultados encontrados não foram estatisticamente significantes $(p=0,534)$, denotando que em média o risco para infecção após inserção de sistema DVP nas cirurgias longas ( $>2 \mathrm{~h}$ ) não foi maior que nas cirurgias de curta duração.

$\mathrm{Na}$ avaliação de derivações neurológicas, o método NNIS, considera todos os procedimentos como limpos ${ }^{13}$. No entanto, ao classificarmos as cirurgias em sistema de DVP do nosso estudo, 91,4\% (53/58) foram limpas, com taxa de infecção de 24,5\% (13/53) o que denota taxa muito elevada.

O índice de risco desenvolvido pelo sistema NNIS infere prognóstico de risco para infecção cirúrgica ${ }^{21}$. Dados de infecção de sítio cirúrgico por procedimento e categoria de risco NNIS de 1992 a 2001, demonstraram taxa de $6,55 \%$ para faixa de risco cirúrgico zero e de $5,21 \%$ para faixa de risco cirúrgico 1 para procedimentos de inserção de sistema de DVP25. Em nosso estudo, a taxa de infecção na faixa de risco zero foi de $25,7 \%$ e nas faixas 1 e 2 de $30,4 \%$, consideradas elevadas principalmente porque a média de risco cirúrgico por paciente foi baixo $(0,41)$. Ao se ajustar à taxa de ISC (superficial, cavidade e órgão) pela média de risco, observa-se taxa também elevada $(58,8 \%)$, indicando que os pacientes apesar de estarem numa faixa de menor risco se infectaram muito. Entre as faixas de risco baixo (zero), intermediária (1) e alta (2) e entre os casos de cirurgia com ou sem infecção de acordo com a categoria de risco não houve diferença estatística no que se refere ao risco de infecção. Esses resultados indicam que a ocorrência de infecção não pôde ser explicada pelos diferentes índices de risco. Estes dados necessitam de construção de curvas de freqüência de infecção para serem cria- 
das possibilidades de intervenção nas práticas preconizadas no serviço.

Taxas de infecção em sistema de DVP de 2,63$3,45 \%$, descritas nas avaliações pelo sistema NNIS de 1992-2004 ${ }^{11}$ diferem do nosso estudo, no qual observamos taxa de infecção cirúrgica de $27,6 \%$ (16/58) por procedimento e de $32 \%$ (16/50) por paciente.

As complicações infecciosas observadas por Jucá et al. ${ }^{26}$ foram ventriculite em $15 \%$ das vezes, seguidas de necrose de pele, deiscência de sutura, coleções subdurais e pseudocistos. As infecções do SNC relatadas por estes autores foram superiores aos resultados por nós encontrados: a meningite associada a ventriculite ocorreu em 5 dos 16 casos de infecção $(31,2 \%)$, o que representou $8,6 \%(5 / 58)$ dos procedimentos. No entanto, freqüências mais baixas de meningite foram relatadas nos estudos de Federico et al. ${ }^{27}$ e de Filka et al. ${ }^{24}$ com $2,6 \%$ e $6,3 \%$ por inserção de cateter em SNC, respectivamente. Embora raro, uma das nossas crianças evoluiu com abscesso cerebral após meningite apesar do tratamento antimicrobiano administrado ${ }^{28}$.

O número de dias de internação pré-operatória como risco de infecção em cirurgia de inserção de sistema de DVP é levantado por Kulkarni et al. ${ }^{3}$, entretanto os resultados não foram conclusivos. Em nosso estudo, a média de internação pré-operatória foi $6,0 \pm$ 8,7 dias no total dos pacientes e $9,4 \pm 12,4$ dias para os casos que evoluíram com infecção, mas sem significância estatística $(p=0,172)$.

A coleta de LCR no pré-operatório com objetivo de excluir infecção prévia do SNC é recomendada por diversos autores ${ }^{5,29}$. Ronan et al. ${ }^{30}$ advertem que o exame de LCR pode apresentar-se normal à microscopia e bioquímica e a infecção ser confirmada somente através da cultura. No nosso estudo, o LCR só foi colhido em 50,0\% (29/58) dos procedimentos o que não permitiu excluir contaminação pré-operatória nos demais casos.

A literatura relata que $70 \%$ das infecções ocorrem até o primeiro mês de pós-operatório e $15 \%$ entre o primeiro e o nono mês ${ }^{7}$, o que está de acordo com os nossos resultados, nos quais $81,3 \%$ (13/16) evoluíram precocemente com infecção de sistema de DVP em menos de 30 dias (média de 26,7 dias).

As infecções de sistema de DVP, caracteristicamente apresentam-se com sinais e sintomas de acordo com o(s) órgão(s) afetado(s). Nossos resultados não diferiram muito dos já descritos por outros autores ${ }^{9,21}$, sendo a febre o sinal clínico mais precoce e freqüente em $81,3 \%$ dos pacientes com infecção (13/16).

No período do seguimento (média de 186 dias) após a alta hospitalar, os 34 pacientes (68\%-34/50) contatados encontravam-se clinicamente estáveis.

É importante enfatizar a necessidade em reduzirmos a freqüência de infecções em DVP em nosso serviço e de se proceder a uma investigação dos possíveis fatores de risco, através de um estudo prospectivo controlado. Em novos estudos, análises dos protocolos de coleta de LCR pré-operatório e de controle de cura nos casos com infecção, avaliação da técnica cirúrgica e tratamento antimicrobiano deverão ser realizados.

Em conclusão, as taxas de infecção por paciente, por procedimento e por índice de risco foram elevadas, mas com média de risco baixa por paciente. Não houve significância estatística entre as variáveis testadas e infecção pós-operatória: tipo de procedimento, idade, tempo de internação pré-operatória, duração da cirurgia, uso de antimicrobiano profilático, uso prévio de cateter de SNC e categoria de risco cirúrgico. A ISC foi a principal complicação infecciosa $(87,5 \%)$ sendo a febre a manifestação clínica mais freqüente $(81,3 \%)$ em menos de 30 dias.

\section{REFERÊNCIAS}

1. Sarguna P, Lakshmi V. Ventriculoperitoneal shunt infections. Indian Med Microbiol 2006;24:52-54.

2. Turgut M, Alabaz D, Erbey F, et al. Cerobrospinal fluid shunt infections in children. Pediatr Neurosurg 2005;41:131-136.

3. Kulkarni AV, Drake JM, Lamberti-Pasculli M. Cerebrospinal fluid shunt infection: a prospective study of risk factors. J Neurosurg 2001;94:195201.

4. Pople IK, Bayston R, Hayward, RD. Infection of cerebrospinal fluid shunts in infants: a study of etiological factors. J Neurosurg 1992;77:29-36.

5. Renier D, Lacombe J, Pierre-Kahn A, Sainte-Rose C, Hirsch JF. Factors causing acute shunt infection: computer analysis of 1174 operations. J Neurosurg 1984;61:1072-1078.

6. Kaufman BA, Tunkel AR, Pryor JC, Dacey RG. Meningitis in the neurosurgical patient. Infect Dis Clin N Am 1990;4:677-701.

7. Tamburrini G, Caldarelli M, Di Rocco C. Diagnosis and management of shunt complications in the treatment of childhood hydrocephalus. Rev Neurosurg 2002;1:26. Disponível em: <http:/ / www.wfns.org/ reviews_neuro3.html>. Acesso em: 05 mar 2004.

8. Choksey, MS, Malik, IA. Zero tolerance to shunt infections: can it be achieved? J Neurol Neurosurg Psychiatry 2004;75:87-91.

9. Bayston R. Hydrocephalus shunt infections. J Antimicrob Chemotherapy 1994;34(Suppl A):75-84.

10. Albright AL, Pollack IF, Adelson PD, Solot JJ. Outcome data and analysis in pediatric neurosurgery. Neurosurgery 1999;45:101-106.

11. CDC NNIS System National Nosocomial Infections Surveillance (NNIS) System Report, data summary from January 1992 through June 2004, issued October 2004. Am J Infect Control 2004;32:470-485.

12. Mangram AJ, Horan TC, Pearson ML, Silver LC, Jarvis WR. Guideline for prevention of surgical site infection 1999;20:217-278.

13. Brasil. Coordenação de Controle de Infecção Hospitalar. NNISS - Vigilância Epidemiológica por Componentes. Brasília, DF: Ministério da Saúde, 1994

14. Gaynes RP, Culver DH, Horan TC, et al. Surgical site infection (SSI) rates in the United States, 1992-1998: The Nacional Nosocomial Infections Surveillance System Basic SSI Risk Index. Clin Infect Dis 2001; 33(Suppl 2):S69-S77. 
15. Culver DH, Horan TC, Gaynes RP, et al. Surgical wound infection rates by wound class, operative procedure, and patient risk index. Nacional Nosocomial Infections Surveillance System. Am J Med 1991;91:152S$157 \mathrm{~S}$

16. Dallacasa P, Dappozzo A, Galassi E, Sandri F, Cocchi G, Masi M. Cerebrospinal fluid shunt infections in infants. Child's Nerv Syst 1995;11: 643-648.

17. Enger PO, Svendsen F, Wester K. CSF shunt infections in children experiences from a population-based study. Acta Neurochir 2003;145: 243-248.

18. Borgbjerg BM, Gjerris F, Albeck MJ, Borgesen SE. Risk of infection after cerebrospinal fluid shunt: an analysis of 884 first-time shunts. Acta Neurochir 1995;136:1-7.

19. Faillace, W. J. Shunt infection. Correspondence. J Neurosurg 2001;94: 1019-1020.

20. Kontny U, Höfling B, Gutjahr P, Voth D, Schwarz M, Schmitt HJ. CSF shunt infections in children. Infection 1993;21:89-92.

21. Morris A, Low DE. Nosocomial Bacterial Meningitis, including central nervous system shunt infections. Infectious Dis Clin N Am 1999;13: 735-750.

22. Camboulives J, Meyrieux V, Lena G. Infections of cerebrospinal fluid shunts in the child: prevention and treatment. Ann Fr Anesth Reanim 2002;21:84-89.
23. Kanev PM, Sheehan JM. Reflections on shunt infection. Pediatr Neurosurg 2003;39:285-290.

24. Filka J, Huttova M, Tuharsky J, Sagat T, Kralinsky K, Krcmery V. Nosocomial meningitis in children after ventriculoperitoneal shunt insertion. Acta Paediatr 1999;88:576-578.

25. Couto RC, Pedrosa TMG. Vigilância Epidemiológica. In Couto RC, Pedrosa TMG (Eds). Guia prático de controle de infecção hospitalar: epidemiologia, controle e terapêutica. 2.Ed. São Paulo: Medsi, 2004:17-62.

26. Jucá CEB, Lins Neto A, Oliveira RS, Machado HR. Tratamento de hidrocefalia com derivação ventriculo-peritoneal: Análise de 150 casos consecutivos no Hospital das Clínicas de Ribeirão Preto. Acta Cirúrgica Brasileira 2002;17(Supl 3):S59-S63.

27. Federico G, Tumbarello M, Spanu T, et al. Risk factors and prognostic indicators of bacterial meningitis in a cohort of 3580 postneurosurgical patients. Scand J Infect Dis 2001;33:533-537.

28. Pandey P, Suri A, Sing AK, Mahapatra AK. Brain abscess: an unusual complication of ventriculo-peritoneal shunt. Indian J Pediatr 2003;70: 833-834.

29. Udani V, Udani S, Merani R, Bavdekar M. Unrecognised ventriculitis/meningitis presenting as hydrocephalus in infancy. Indian Pediatrics 2003;40:870-873.

30. Ronan A, Hogg GG, Klug GL. Cerebrospinal fluid shunt infections in children. Pediatr Infect Dis J 1995;14:782-786. 